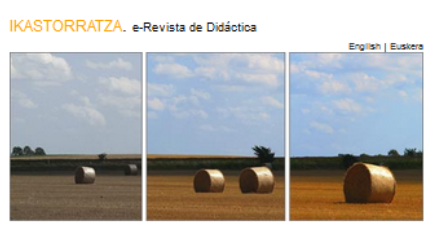

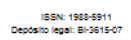

Inicio, Sobre nosotros, Publicacionesı, Participa

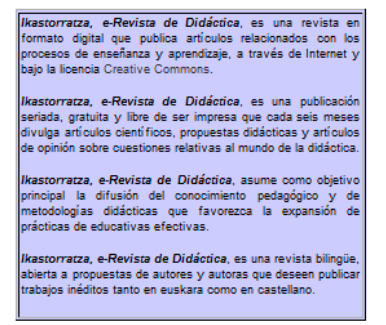

\section{IKASTORRATZA. Didaktikarako e-aldizkaria}

\author{
IKASTORRATZA. e-journal on Didactics
}

\title{
Aprendizajes informales. Perspectivas teóricas y relatos de aprendizajes
}

Rocío Belén Martín rbmartin@unc.edu.ar

Danilo Silvio Donolo

donolo@gmail.com

To cite this article:

Martín, R. B.. \& Donolo, D. S. (2019). Aprendizajes informales. Perspectivas teóricas y relatos de aprendizajes. IKASTORRATZA. e-Revista de Didáctica, 23, 115-131. DOI: 10.37261/23_alea/5

To link to this article:

https://doi.org/10.37261/23_alea/5

Published online: 30 December 2019 


\title{
Aprendizajes informales. Perspectivas teóricas y relatos de aprendizajes
}

\author{
Rocío Belén Martín \\ Facultad de Ciencias Exactas, Físicas y Naturales, Universidad Nacional de Córdoba \\ CIT Villa María CONICET \\ rbmartin@unc.edu.ar
}

Danilo Silvio Donolo

Universidad Nacional de Río Cuarto

CONICET

donolo@gmail.com

\section{Resumen}

Este artículo tiene como propósito profundizar en desarrollos teóricos y empíricos sobre el aprendizaje informal. El aprendizaje informal es un tópico que refiere al aprendizaje de las personas a través de diversos entornos sociales: en el hogar, en actividades con amigos, viajes, centros comunitarios, iglesias, etc. El escrito comienza con una pequeña revisión exploratoria sobre el término y sus variaciones, y luego se realiza una recopilación de relatos sobre algunos ejemplos de aprendizaje informal. En este intento, Ciencias, TIC, Trabajo, Artes, Relaciones Sociales y Habilidades para la Vida podrían ser pensados como formas de aprendizaje informal. La variedad de construcciones para llamar al aprendizaje, la multiplicidad de intentos de definirlo, y configurarlo, son importantes para re-construir y desarrollar nuevos marcos teóricos sobre el concepto en el campo de la Psicología Educacional.

Palabras clave: Aprendizaje informal, contextos, TIC, Educational Psychology.

\section{Abstract}

The purpose of this article is to deepen theoretical and empirical developments about informal learning. Informal learning is a subject that translates into the learning of people through various social media: at home, activities with friends, trips, community centers, churches, etc. its variations, and then there is compilation of stories about some examples of informal learning. In this attempt, Science, ICT, Work, Arts, Social Relations and Life Skills could be thought of as forms of informal learning. The variety of constructions to call learning, the multiplicity of attempts to define it, and to configure it, are important to re-construct and develop new theoretical frameworks on the concept in the field of Educational Psychology.

Key Words: Informal learning, contexts, ICT, work, Psicología Educacional. 


\section{Introducción}

En el campo de la Psicología Educacional, se vienen utilizado diferentes significaciones para nombrar a los espacios y formas de aprendizaje; Aguirre y Vázquez (2004) en uno de sus escritos hablan de 'situaciones educativas', y distinguen tres tipos: formal, no formal e informal, añadiendo que dichas situaciones presentan características especiales que las diferencian. En un contexto ibérico (Sarramona, Vázquez y Colom, 1998; Trilla, Gros, López y Martín, 2003) utilizan el término ‘educación’ aludiendo en sentido más amplio para referirse a estas situaciones. A diferencia de escritos, con una impronta más anglosajona (Colardyn y Bjornavold, 2004; Sequel Project, 2004; Cobo y Moravec) utilizan el término ‘aprendizaje' para referir en sentido amplio a estos contextos aprendizaje formal, aprendizaje no formal y aprendizaje informal- (Martín, 2014; Martín, Rinaudo y Paoloni, 2019).

Rinaudo (2019) expone que la delimitación de esta nueva línea de estudios fue posible por ciertos cambios experimentados en el campo de la Psicología Educacional; por un lado, un alejamiento de las perspectivas que privilegiaban a las personas como las principales unidades de análisis en el estudio de los aprendizajes; en esto, el progresivo corrimiento hacia los enfoques socioculturales. Por otro lado, también bajo el influjo de las perspectivas socioculturales, se dieron avances significativos en torno de la noción de contexto, que deja de referirse exclusivamente a los contextos escolares, entendidos muchas veces como contenedores cerrados, para incluir las oportunidades de aprendizaje que se generan fuera de la escuela, muy particularmente en los hogares, centros comunitarios, clubes, bibliotecas, museos, iglesias, entre otros (Barron y Bell, 2016; Rinaudo, 2019).

El escrito que aquí se presenta, inicia con una pequeña revisión exploratoria sobre el término y sobre concepciones similares, en continuidad se realiza una recopilación de relatos sobre las formas que va tomando el aprendizaje informal.

\section{Aprendizaje informal, algunas consideraciones...}

Para comenzar con este apartado, es preciso explicitar que aquí no se pretende elaborar un estado del arte completo y documentado acerca del tema, interesa mostrar las ideas acerca del aprendizaje informal que tienen fuerte impacto en la investigación educativa. Es posible vislumbrar múltiples intentos por definir al aprendizaje informal, desde la 
Pedagogía, Antropología y la Psicología Educativa recientemente se encuentran diferentes definiciones.

Más focalizado en los procesos cognitivos que en los ambientes de aprendizaje, Schugurensky (2000), propone tres formas de aprendizaje informal utilizando criterios como la intencionalidad y la conciencia:

1) Autodirigido: refiere a un aprendizaje consciente e intencional, que se desarrolla de forma individual o como parte de un grupo, sin la ayuda de un facilitador.

2) Incidental: es un aprendizaje no intencional, pero la persona es consciente de la experiencia que se produce cuando él no tiene ninguna intención previa de aprender algo.

3) Socialización: es inconsciente y no intencional, precisamente cuando las personas interiorizan nuevas actitudes, habilidades, comportamientos, etc.

El aprendizaje informal, es entendido como el que acontece indiferenciada y subordinadamente a otros procesos sociales; es decir, cuando se está inmerso en otras realidades culturales (Trilla, 1992). El concepto aprendizaje informal, fue introducido por Knowles en Informal Adult Education (1950), pero asimismo, tanto John Dewey como Mary Parker Follett desde comienzos del siglo XX habían fomentado prácticas de aprendizaje informal (Transliteracy, 2018).

En este sentido, Asenjo, Asensio y Rodríguez-Moneo (2012) postulan que el término es complejo y un tanto confuso debido a la falta de consenso, con interpretaciones diversas. En su escrito mencionan que uno de los trabajos pioneros, sobre la descripción del aprendizaje informal fue el publicado en el año 1974 por Charles Screven, surgiendo -el aprendizaje informal- a partir de los intentos de distanciar este concepto con el de aprendizaje no formal; en la medida en que en los contextos de presentación del patrimonio comienza a usarse, no sólo para denominar procesos de aprendizaje, sino también para denominar las características que deben conllevar los procesos de enseñanza para fomentar la participación activa y el control sobre su propio aprendizaje cuando se desarrolla fuera de instituciones educativas formales.

Cuando se habla de aprendizaje informal se hace referencia a cuando las personas aprenden a través de diversos entornos sociales, exceptuando y sin hacer énfasis en lo escolar, como en el hogar, en actividades con amigos, en viajes, en museos, en potencialmente todos los lugares donde experimentan y asumen nuevas búsquedas. En los contextos informales las personas pueden desarrollar conciencia, interés, motivación, competencias sociales y prácticas, e identidades en su trayectoria de aprendizaje. Las 
conexiones entre experiencias, capacidades y nuevas oportunidades para aprender continúan a lo largo de la vida de una persona (Bell, Lewenstein, Shouse y Feder, 2009). Otros autores (Alves da Silva y Ferreira, 2016) sostienen que el "aprendizaje informal es el proceso por el cual las personas adquieren los conocimientos, habilidades y actitudes con la experiencia cotidiana y la exposición al medio ambiente en el que viven (2016:8), ocurriendo dondequiera que las personas tengan la necesidad, la motivación y oportunidad para el aprendizaje ya sea la escuela misma, el hogar o el club de barrio.

Livingstone (1999 en Schugurensky, 2000) considera que el aprendizaje informal puede definirse como una actividad que involucra la búsqueda de comprensión, conocimiento o habilidades que ocurre fuera los planes de estudio de las instituciones educativas, o los cursos o talleres ofrecidos por agencias educativas o sociales. En decir, el aprendizaje informal incluye al aprendizaje que se produce fuera del currículo formal y no formal, de instituciones y programas educativos. En este sentido, nos parece importante recuperar una reflexión que realiza Schugurensky (2000) sobre la utilización de la palabra "aprendizaje":

Es importante tener en cuenta que estamos utilizando deliberadamente la palabra "aprendizaje" y no "educación", porque en los procesos de aprendizaje informal no hay instituciones educativas, instructores institucionalmente autorizados o currículos prescritos. También es pertinente tener en cuenta que estamos diciendo 'fuera de los planes de estudio de las instituciones educativas' y no 'fuera de instituciones educativas', porque el aprendizaje informal también puede tener lugar dentro de instituciones educativas no formales. En ese caso, sin embargo, los aprendizajes ocurren independientemente (y en ocasiones en contra) de los objetivos previstos del plan de estudios explícito (Schugurensky, 2000:2) ${ }^{1}$

En lo que respecta a estos aprendizajes, los sesgos cognitivos influyen y/o favorecen que las personas se autoafirmen en sus creencias. Aportes en esta línea son los introducidos por Kanheman (2011) psicólogo que estudia sobre el juicio y la toma de decisiones, desde el abordaje de su teoría acerca de las perspectivas (de Pablo, 2005).

1 La traducción es nuestra. 
En este sentido, nos parece importante recuperar algunas concepciones que aparecen ligadas al aprendizaje informal, con definiciones similares y contextos de origen y producción diversos, son: el aprendizaje permanente y/o aprendizaje a lo largo de la vida, entornos personales del aprendizaje (PLE), ecologías de aprendizaje, aprendizaje invisible y contextos híbridos.

Dávila Heitman (2013) considera que la idea de aprendizaje a lo largo de la vida nace de la concepción de la educación permanente, que a través del curso del tiempo va adquiriendo diferentes particularidades. El concepto, surge en los años 70' como una alternativa a la educación escolar, cuestionada por autores como Illich (1971) y Reimer (1970), quienes que consideraban que limitaba la curiosidad, la iniciativa, la autonomía, la creatividad y el pensamiento crítico, reforzando la sumisión y el adoctrinamiento de los estudiantes.

Bell, Lewenstein, Shouse y Feder (2009), realizan una detallada diferenciación y plantean la idea del aprendizaje permanente (lifelong learning), a lo largo de toda la vida (life-wide learning) y de la vida (loofe-deep learning) ha sido influyente en esfuerzos por desarrollar una noción amplia de aprendizaje, incorporando cómo las personas aprenden sobre el curso de la vida, a través de las configuraciones sociales, y en relación con el predominio de las influencias culturales.

Cuando los autores mencionan el aprendizaje permanente, refieren más a la adquisición de competencias, actitudes y a una habilidad sobre el uso de información respecto al curso de la vida, reconociendo que las necesidades de desarrollo y los intereses varían en diferentes etapas de la vida. En general, las personas prefieren buscar información y adquirir formas de hacer las cosas de acuerdo a sus intereses, necesidades, curiosidad, placer y sentido teniendo talentos que se alinean con ciertos tipos de tareas y desafíos. Si bien es menester aclarar que actualmente estamos inmersos en un proceso de polarización ideológica que se produce en esta interacción contante en y con las redes sociales, y que amerita un estudio particular en relación al aprendizaje informal y la construcción de la opinión pública en procesos electorales ${ }^{2}$.

El aprendizaje a lo largo de toda la vida, se vincula al aprendizaje que se lleva a cabo rutinariamente, a través de diversos entornos sociales, actividades áulicas, programas

\footnotetext{
2 Trabajos interesantes en esta línea son los de Robert Kaufman sobre gobernabilidad y polarización ideológica.
} 
extracurriculares, instituciones educativas informales, lugares en línea, hogares y otros locales de la comunidad. El aprendizaje se desarrolla, tanto en las oportunidades y patrones, a partir de la amplitud de experiencias humanas y se relaciona en los apoyos y ocasiones para el aprendizaje que están disponibles para un individuo o grupo. Los estudiantes deben aprender a navegar por las diferentes suposiciones subyacentes y los objetivos asociados con la educación y el desarrollo.

El aprendizaje de la vida se refiere a creencias, ideologías y valores asociados con ir viviendo la vida y participando en el funcionamiento cultural de las comunidades y de una sociedad más amplia. Tal aprendizaje refleja la moral, la ética, lo religioso y los valores sociales que guían lo que las personas creen, cómo actúan y cómo juzgarse a sí mismos y a los demás. Este enfoque en el aprendizaje a lo largo de la vida enfatiza cómo el aprendizaje nunca es un esfuerzo sin cultura, por ejemplo, el aprendizaje de valores para vivir en sociedad, lo que implica normas de convivencia.

Tomados en conjunto, estos conceptos ponen de manifiesto la amplitud del aprendizaje humano y enfatizan sobre el amplio alcance de los entornos informales. En estos entornos, el aprendizaje se logra a través de múltiples configuraciones, de recursos materiales y sociales cambiantes, en la variedad de formas que las personas participan y hacen uso de sus conocimientos, sus diversas redes sociales, agrupaciones, sus propósitos y expectativas en evolución.

Los entornos personales de aprendizaje, ecologías de aprendizaje y aprendizaje invisible son tres concepciones que están más ligadas a las TIC. Por entornos personales de aprendizaje (Personal Learning Environments -PLEs-), se entienden a aquellos espacios que son especialmente interesantes en el marco de una sociedad basada en el conocimiento que exige que el aprendizaje sea una actividad constante a lo largo de toda la vida. Aprendemos informalmente de manera constante, en diferentes grados y matices, y en variados contextos; en el trabajo, en el hogar, en grupos primarios de socialización, en el cine, leyendo un libro o en los propios centros educativos, dentro y fuera de las aulas. Los PLEs son el término con el que se denomina a un entorno de red en el que, junto a los objetos y espacios físicos y los contactos personales, desarrollamos nuestras vidas; del mismo modo que leemos un libro, hablamos con un colega, asistimos a una charla en un congreso, leemos y escribimos en blogs, participamos en debates o recibimos por email la recomendación de un colega de no perdernos un vídeo subido a YouTube (Adell, 2010). 
Las ecologías de aprendizaje, en cambio, son definidas por Seely Brown (1999) ${ }^{3}$ como sistemas abiertos, dinámicos e interdependientes, con la organización de diversos agentes. Parcialmente son concebidas como auto-aprendizaje, con carácter adaptativo y, a la vez, frágil. Un trabajo interesante y reciente sobre ecologías de aprendizaje y educación expandida, es la compilación que realizan Martínez Rodríguez y Fernández Rodríguez (2018), atendiendo a la investigación acerca de los procesos de construcción del conocimiento, la generación de saberes y la adquisición de aprendizajes desde cuatro dimensiones: en tanto ecologías personales del aprendizaje, ecologías de recursos, ecologías comunitarias y ecologías de saberes.

El aprendizaje invisible, es definido por Cobo y Moravec (2011) como una propuesta que procura integrar diversos enfoques en relación con un nuevo paradigma de aprendizaje y desarrollo del capital humano. Considerando el impacto de los avances tecnológicos y las transformaciones de la educación formal, no formal e informal, como aquellos metaespacios intermedios. En su libro, los autores hacen referencia a diferentes aprendizajes y redes para aprender como twitter, facebook, entre otras. En una entrevista realizada a Reig (2011), destaca algunos ejemplos como el proyecto de Educación Expandida del colectivo Zemos 98 (http://zemos98.org/); más cercano al discurso de la educación informal. Añadiendo que las comunidades de práctica se enmarcan en este movimiento, con posibilidades de crear grupos en la web o entornos de colaboración que amplían el ámbito del aprendizaje social (del Moral, 2015; Fernández Sánchez y Valverde Berrocoso, 2014; Sanz Martos, 2005).

Éstos últimos tres conceptos tienen un tema aglutinador, el énfasis en el aprendizaje mediado por TIC, el uso de las tecnologías en la vida cotidiana de la persona. Con esto, no podemos desconocer que en el tema del aprendizaje informal, si bien el ser humano tiene un carácter social como especie, el término fue adquiriendo difusión y crecimiento, y encuentra una estrecha relación con las tecnologías de la información y comunicación. Otro tipo de concepto muy relacionado con las ecologías de aprendizaje y el aprendizaje informal, esencialmente, es el de 'contextos híbridos', entendidos como contextos donde hogares, escuelas y comunidades se integran para proporcionar mayores oportunidades de aprendizaje. Los estudios sobre contextos híbridos son aquellos que no consideran al aprendizaje sólo en ambientes escolares, sino que también lo contemplan en su relación

3 John Seely Brown es un investigador que se especializa en estudios organizacionales con una inclinación particular hacia las implicaciones organizativas de las actividades asistidas por computadora. 
con ámbitos como clubes, centros comunitarios, parques, lugar de trabajo, hogares, iglesias, entre otros (Barron y Bell, 2016). Hacen foco en diferentes modos de participación y permiten delinear diferentes trayectorias de aprendizaje, presentándose como una posibilidad para proporcionar mayores oportunidades de aprendizaje a partir de la integración de diversos entornos (Barron y Bell, 2016). El aprendizaje en éstos contextos, puede ser "por ejemplo, un estudiante puede tener aprendizajes formales en su casa (hacer un tarea o trabajo, por ejemplo) o tener un aprendizaje informal en el colegio (aprendiendo con sus compañeros un nuevo truco para utilizar un videojuego)” (Cobo y Moravec, 2011: 120).

Variados autores, en intentos de hacer una tipología, mencionan que el aprendizaje informal, se da a lo largo de la vida cotidiana con prácticas relacionadas con el trabajo, la familia y el ocio (Coladyn y Bjornavold, 2004).

\section{Tipos, formas, contextos y variaciones en los aprendizajes informales}

En el marco de estudios previos (Martín, 2019; Martín y Corradini, 2019), pensamos en una categorización sobre las formas de aprendizaje informal haciendo alusión a contenidos, contextos, entre otros aspectos. En este intento, Ciencias, TIC, Trabajo, Artes, Relaciones Sociales y Habilidades para la Vida podrían ser pensados como contextos donde pueden identificarse formas de aprendizaje informal.

Los aprendizajes informales sobre Ciencias y TIC son los que aparecen más documentados (Bell, Lewenstein, Shouse y Feder, 2009; Asenjo, Asensio y RodríguezMoneo, 2012; Crowley, Pierroux y Knutson, 2014; Ibáñez Etxeberria, Vicent Otaño y Brouard, 2012). Algunos de los trabajos que se encuentran refieren al aprendizaje informal con dispositivos móviles, en museos virtuales, con trabajos de laboratorios, relativos a patrimonio, entre otros.

Los aprendizajes sobre el Trabajo implican una amplia variedad de maneras improvisadas, no oficiales y espontáneas, en la que las personas aprenden en su trabajo, y esas prácticas de aprendizaje, que en algunos casos se retroalimentan en el trabajo colaborativo. Por ejemplo, en la mayoría de las organizaciones, en las cooperativas, existe una estructura formal descrita por los organigramas, documentos y procedimientos, y un ambiente informal basado en las relaciones y auto-organización de grupos de personas, es decir, una red informal de personas que trabajan juntas compartiendo conocimientos, resolviendo problemas e intercambiando ideas, relatos y confidencias (Adobe, 2007). O bien, un aporte en este sentido es el trabajo de Lin y Bound (2011) quienes realizaron una 
investigación en el contexto de formación de chefs, y observaron que tanto los supervisores como los colegas proporcionaban recursos interpersonales e informacionales que ayudaban a aprender a quienes se estaban capacitando y así progresivamente iban desplegando identidades profesionales (Martín, 2017).

Para ilustrar las formas de aprendizaje nos interesa recuperar algunas apreciaciones de personas acerca sus aprendizajes más importantes, elegimos iniciar las conversaciones con 20 personas, de forma exploratoria mediante Whatsapp ${ }^{4}$ (en audio y texto). En este sentido, Scribano (2017) expone que en Whatsapp se perciben y experimentan elaboraciones múltiples de los límites de lo real, la aplicación conlleva en la práctica, agudizar la mirada y des-estructurar la naturalización de lo que hay en todos los días. En su escrito, además de exponer una experiencia utilizando Whatsapp, invita a profundizar en la evaluación de nuevas modalidades de indagación en Ciencias Sociales.

El sistema de mensajería de WhatsApp es un tipo de lenguaje virtual instantáneo, en la categoría de lo hablado escrito, pensado para teléfonos móviles inteligentes. Es un sistema que comparte algunas peculiaridades de otras técnicas de comunicación electrónicas pero que aporta otros elementos novedosos como el código iconográfico emoji (Calero Vaquera, 2014).

Creemos que para hablar del aprendizaje informal en relación a Trabajo, Ciencia y TIC, Luis ${ }^{5}$ podría ser un buen ejemplo.

Yo uso para aprender, leer o demás, uso muchos blogs de investigación, por ejemplo hay unos links que se llaman Research Gate, que vos te logueas ahí y tenes investigadores de todo el mundo, protocolos, experimentales, concentraciones, $\mathrm{y}$ después muchos papers, yo todo el tiempo leo trabajos de investigación para informarme de lo que sea (Luis).

Otro tipo de aprendizaje informal muy recurrente, y que es común, es el relativo a las Artes, tales como: arquitectura, escultura, pintura, música, poesía/literatura, fotografía y el cine. La educación puede ser a través de las artes, teniendo como propósito fortalecer por medio de las artes y la cultura, la adquisición de habilidades humanas en general, la formación en valores y la respuesta frente al estudio de áreas que no son artísticas. O bien,

4 Whatsapp: es una aplicación de mensajería para teléfonos inteligentes, que envía y recibe mensajes mediante Internet. https://es.wikipedia.org/wiki/WhatsApp

5 Luis, 34 años, investigador y profesor. Está preocupado por conocer más terapias para el cáncer. 
la educación en las artes puede buscar favorecer el desarrollo de habilidades propiamente artísticas y culturales (Consejo Nacional de la Cultura y las Artes, 2016).

Para el aprendizaje de las Artes recuperamos un fragmento del relato de Arabela ${ }^{6}$

... ¿cómo puede ser que no se me ocurran otros aprendizajes para relatar? Y allí pensé en cómo se aprende a tocar el piano. Cuando era niña fui a una academia en donde enseñaban teclado, y luego, de más grande, tomé algunas clases en conservatorio. En estos contextos, aprendí cuestiones relativas a cómo leer partituras. Pero luego, por muchos años dejé de hacerlo. Hace unos meses, comencé a intentar tocar nuevamente y para ello busqué algunas nuevas partituras. Así que considero que este es un aprendizaje importante, el que realizo cuando intento 'sacar' una partitura y tengo que 'leer' las notas, repasar algunas cuestiones, buscar o averiguar qué significa un símbolo que aparece en la partitura... Además, se suma el aprendizaje del uso de un nuevo instrumento (ya que el acercamiento actual al instrumento es a partir de un nuevo piano digital, con características muy diferentes a aquel que tocaba cuando era niña). En ese sentido, aprendo continuamente también acerca de las nuevas posibilidades que esta nueva herramienta me da: manejar la sensitividad de las teclas, utilizar el pedal de sustain, configuración de funciones como metrónomo, conexión del piano a otros parlantes (cuestiones que -por supuesto- aquel primer teclado que me trajo Papá Noel en 1991 no tenía!) (Arabela).

Esto nos lleva a pensar que ante la elección de una carrera orientada a las Artes, mayoritariamente el aprendizaje se comienza en contextos informales o no formales, o bien como dice Arabela se continúa en ellos para seguir aprendiendo.

En tanto Gabriel ${ }^{7}$, sus obras fueron calando hondo y forman parte de un blog con publicaciones compartidas, sus aprendizajes sobre la poesía siguen manteniéndose en entornos informales, compartidos con poetas, formando parte de una comunidad de práctica de poetas puntanos.

El aprendizaje de y a través de relaciones sociales es una forma de aprendizaje informal poco consciente e intencional pero muy común; aprender con otros, a partir de los vínculos y la socializaciones son formas de las que aprendemos todos los días.

6 Arabela, 35 años, pianista y profesora.

7 Gabriel: http://revistaculturalelviento.blogspot.com/2010/08/gabriel-rosales-poeta-de-el-viento.html 
El caso de Ana ${ }^{8}$, que para el aprendizaje de su trabajo, revaloriza las relaciones sociales, la importancia de las redes y comunidades. El aprendizaje con los otros.

[27/9 20:06] Anita: Casi no recuerdo como era el aprendizaje formal, porque hace años que uso el informal

[27/9 20:07] Anita: Mucha búsqueda de información a través de buscadores sobre lo que me va interesando, algunas veces motivada solo por el mero conocimiento y la gran mayoría para ir resolviendo algún desafío puntual. Videos, muchos, sobre todo para procedimientos más técnicos. En cualquier idioma, si puedo uso el subtitulado de YouTube para algunos detalles. Cuando encuentro varias opciones, y ninguna me cierra del todo o no coincide, ensayo y error pero siempre de acuerdo a lo que fui investigando antes

[27/9 20:11] Anita: Estoy en varios foros y comunidades de técnicos, eso más que nada para temas más puntuales, aprendo muchísimo leyendo comentarios de usuarios que tienen más experiencia, incluso aunque no sea un tema planteado por mí, lo sigo para ver cómo se resuelve, y como realizan otras personas el procedimiento

[27/9 20:15] Anita: Y un poco de ayuda entre colegas presenciales

[27/9 20:21] Anita: Ah, un par de talleres online que se vienen haciendo, también de allí he sumado aprendizaje, pero por cuestiones de horario, los he visto luego de emitidos online en canales de YouTube. Voy a intentar estar en alguno porque se pueden ir haciendo preguntas en vivo

[27/9 20:30] Anita: Jajajaja es que si tengo que decir un hobbie o algo que me gusta hacer, es aprender (Ana)

Como en el caso de Luis, Ana también usa el aprendizaje de las TIC en su trabajo, con esto no queremos hacer una división, ni compartimentar el aprendizaje informal, sino aproximarnos a las formas, sus conexiones, características y confluencias.

A esto le podemos agregar lo que nos cuenta Amaro9: "escucho ideas, aprendo, trato de formar mi propia ideología”, sus aprendizajes, discurren en la secundaria entre amigos, charlas y con su guitarra.

8 Ana, 43 años, técnica informática, tiene como hobby aprender.

9 Amaro, 17 años, estudiante de Secundario, arquero, guitarrista y dibujante. 
En cuanto al aprendizaje de habilidades cotidianas pensamos en la cocina, y otras habilidades para la sostenibilidad de la vida. Cecilia ${ }^{10}$ cuenta como el aprendizaje de la cocina se va mediando en las relaciones sociales, y lo que significa cocinar en comunidad, en donde el papel de las madres, los padres y familiares aparecen con más énfasis.

Mmm en la infancia? Las carneadas en el campo. Las hacíamos cuando era chica. Eran en las vacaciones de julio. Pleno invierno, toda cada integrante de la familia tenía un rol. Nos levantamos temprano y duraban más de 2 días. Era una fiesta porque además venía otra gente a ayudar y se comía muy rico. Mi tarea era cortar grasitas para hacer chicharrones y cortar carne para los chorizos. Creo que ahí aprendí a diferenciar el olor de las especies, a ver cómo y cuáles son las distintas partes de los animales, de que se alimentaban, cuál era el mejor alimento, a cocinar, ver cómo es el proceso de elaboración de los productos. Y a comprender cómo todo sirve: las tripas, la sangre, el cuero, la grasa, etc. Además era lindo momento familiar, al lado del fuego, los almuerzos largos, la gente que venía no cobraba sino que era bueno regalar carne, chorizos, etc. (Cecilia)

\section{Consideraciones finales}

A modo de cierre de este escrito, pensamos que existen algunos aspectos interesantes para pensar en los aprendizajes informales, uno de ellos es atender al aprendizaje en la vida y no para la vida, nos gusta más esta apreciación porque vamos aprendiendo múltiples cosas, situaciones y habilidades en el transcurrir de la vida. Otra apreciación es que nos parece recomendable seguir profundizando es el desarrollo y las formas que adquieren los procesos de aprendizaje en la multiplicidad de contextos. Por ello, atendiendo al aprendizaje que ocurre en la vida, quisimos recuperar relatos de personas en sus aprendizajes informales, con estilos de narración más informal, siendo una tarea difícil formalizar lo informalizado.

Mayoritariamente, y esto llamo nuestra atención, que cuando se habla de aprendizaje informal, de aquel aprendizaje fuera de la escuela, existe cierta nostalgia, y retrotrayéndose a los aprendizajes acontecidos en la infancia. En esta dirección nos preguntamos ¿̇las personas son conscientes que siguen aprendiendo? Se aprende del y en el trabajo, de las relaciones sociales, hay diferentes ámbitos, y personas que participan en nuestras experiencias de aprendizaje ¿las percibimos?

10 Cecilia, 33 años, investigadora que se ocupa de trabajar sobre la política habitacional de los pueblos indígenas. 
En esto de revalorizar el aprendizaje informal en contextos formales, aparecen diversidad de estudios que muestran que el aprendizaje no es un concepto que pueda clasificarse o delimitarse, sino que se inscribe en las prácticas, como lo es el concepto de contextos híbridos, donde se desdibujan los límites entre los espacios de aprendizaje formal e informal (Barron y Bell, 2016). Remarcándose que es cada vez más relevante la disonancia que existe entre lo que aprenden los jóvenes en el salón de clases y en su vida cotidiana, ya que la escuela encuentra dificultades en acompañar esos tránsitos y evoluciones (Pereira, Fillol y Moura, 2019). Del mismo modo que postula Schugurensky (2000) el aprendizaje informal puede acontecer independientemente dentro de las instituciones educativas, aunque no esté contemplado dentro de los planes de estudio. Van apareciendo nuevos ambientes de aprendizaje, nuevos escenarios, que buscan diversificar la oferta formativa (Salinas, 1997), que ponen de relieve el carácter situado de nuestro aprendizaje, ya que es resultado de los variados escenarios en que participamos, cada uno de los cuales otorga oportunidades y herramientas para aprender (Coll, 2013). Por ello, esta diferenciación que hacemos entre aprendizajes no es con una intención, ni fines taxonómicos, sino que invitamos a pensar diferentes contextos de aprendizajes. En este sentido, pensamos en la variedad de maneras de llamar al aprendizaje, la multiplicidad de intentos de definirlo, configurarlo, empiezan a ser considerados en diversos estudios son un tema de agenda actual (Barron y Bell, 2016; Rinaudo, 2019). Además que la importancia en considerar nuevas metodologías para el aprendizaje, de lo que ocurre en todos los días (Scribano, 2017).

Burbules (2012) sostiene que se requieren nuevas teorías del aprendizaje que integren el aprendizaje formal, informal y el aprendizaje experiencial y situado, y del mismo modo, se requieren habilidades en el diseño de estrategias de aprendizaje que aprovechen e interrelacionen el aprendizaje que tiene lugar en contextos diversos potenciando los elementos claves que dan en los procesos de aprendizaje. 


\section{Referencias}

Adell, J. y Castañeda, L. (2010). Los Entornos Personales de Aprendizaje (PLEs): una nueva manera de entender el aprendizaje. En Roig R. Vila y M. Fiorucci, (Eds.), Claves para la investigación en innovación y calidad educativas, La integración de las Tecnologías de la Información y la Comunicación y la Interculturalidad en las aulas. Alcoy, Marfil.

Adobe Systems Incorporated (2007). Informal Learning: Extending the impact of Enterprise ideas and information. A conversation with Jay Cross.

Aguirre Pérez, C. y Vázquez Molini, A. (2004). Consideraciones generales sobre la alfabetización científica en los museos de ciencia como espacios educativos no formales. Revista Electrónica de Enseñanza de las Ciencias, 3 (3), 1-26.

Alves da Silva, C. y Ferreira, C. (2016). Las redes sociales y el aprendizaje informal de Estudiantes de Educación Superior. Acción Pedagógica, 25, 6-20.

Asenjo, E., Asensio M. y Rodríguez-Moneo, M. (2012). Apredizaje Informal. En M. Asensio, E. Rodriguez, Asenjo y Castro (Eds.), SIAM. Series Iberoamericanas de Museología, Vol. 2.

Barron B. y Bell, P. (2016). Learning enviroments in and out of school. En L. Corno y E. Anderman, Handbook of Educational Psychology. Third Edition (pp. 323-335). New York: Routledge.

Bell, P., Lewenstein, B., W. Shouse, A. y A. Feder, M. (2009). Learning science in informal environments. People, places, and pursuits, $1^{\circ}$ edición. Washington: D.C., The National Academic Press.

Brown, S. (1999). Sustaining the Ecology of Knowledge. Spring, 12.

Burbules, N. (2012). El aprendizaje ubicuo y el futuro de la enseñanza. Encounters on Education, 13.

Calero Vaquera, M. L. (2014). El discurso del WhatsApp: entre el Messenger y el SMS. Oralia, 17, 85-114.

Cobo Romaní, C. y Moravec J. (2011). Aprendizaje Invisible. Hacia una nueva ecología de la educación, Colección Transmedia XXI. Laboratori de Mitjans Interactius. Barcelona: Universitat de Barcelona.

Colardyn, D. y Bjornavold, J. (2004). Validation of Formal, Non-Formal and Informal Learning: policy and practices in EU Member States. European Journal of Education, 39 (1), 69-89. DOI: https://doi.org/10.1111/j.0141-8211.2004.00167.x 
Coll, C. (2013). El currículo escolar en el marco de la nueva ecología del aprendizaje. Aula de innovación educativa, 219, 31-36.

Consejo Nacional de la Cultura y las Artes (2016). Cuaderno 2: Por qué enseñar arte y cómo hacerlo. Caja de herramientas para la educación artística.

Crowley, K., Pierroux, P. y Knutson, K. (2014). Informal Learning in Museums. En R.K Sawyer (Ed.), The Cambridge Handbook of the LearningSciences. New York, Cambridge University Press.

Dávila Heitman, D. (2013). Aprendizaje a lo largo de la vida. Antecedentes y desafíos para la universidad de hoy. Ciencia y Cutura, 30, 87-101.

De Pablo, C. (2005). Después de Kahneman y Tversky: ¿Qué queda de la Teoría Económica?. Revista de Economía y Estadística, 43 (1), 55-98.

del Moral Pérez, M. E. (2015). Comunidades virtuales de videojugadores: Comportamiento emocional y social en poupée girl. Revista De Educación a Distancia, (33).

Fernández Sánchez, M. R. y Valverde Berrocoso, J. (2014). Comunidades de práctica un modelo de intervención desde el aprendizaje colaborativo en entornos virtuales. Comunicar. Revista científica iberoamericana de comunicación y educación, 42, 97-105.

Ibáñez Etxeberria, A., Vicent Otaño, N. y Asensio Brouard, M. (2012). Aprendizaje informal, patrimonio y dispositivos móviles. Evaluación de una experiencia en educación secundaria. Didáctica de las Ciencias Experimentales y Sociales, (26), 3-18.

Kanheman, D. (2011). Pensar rápido, pensar despacio. Editorial Debate.

Martín R. y Corradini M. (2019). La construcción de noticias en comunidades de aprendizaje. El caso de un taller de producción gráfica. Revista Magis, 11 (23). DOI: 10.11144/Javeriana.m11-23.cnca

Martín, R. B. (2019). Perspectiva teórica sobre el estudio de los contextos y comunidades de aprendizaje. En Martín, R., Rinaudo, M. C. y Paoloni, P. V., Comunidades. Estudios y experiencias sobre contextos y comunidades de aprendizaje (pp. 75 131). Villa María: Eduvim.

Martín, R. B. (octubre, 2018). ¿Por qué estudiar las cooperativas? Reflexiones sobre las cooperativas como contextos de aprendizaje y trabajo. III Congreso Internacional y VI Congreso Nacional de la Psicología, Córdoba. 
Martín, R. B. (2017). El aprendizaje de la identidad profesional. Expresiones sobre el aprendizaje para el trabajo. Anuario de Investigaciones de la Facultad de Psicología, 3 (2).

Martín, R. B. (2014). Contextos de aprendizaje formales, no formales e informales. Ikastorratza e-Revista de didáctica, 12.

Martín, R., Rinaudo, M. C. y Paoloni, P. V. (2019), Comunidades. Estudios y experiencias sobre contextos y comunidades de aprendizaje. Villa María: Editorial Eduvim.

Martínez Rodríguez, J. B. y Fernández Rodríguez, E. (2018), Ecologías de Aprendizaje: Educación Expandida en Contextos Múltiples. España: Ediciones Morata, S.L.

Pereira, S., Fillol, J. y Moura, P. (2019). El aprendizaje de los jóvenes con medios digitales fuera de la escuela: De lo informal a lo formal. Revista Comunicar, XXVII (58). DOI: https://doi.org/10.3916/C58-2019-04

Reig, D. (2011). Vox populi e in-conclusiones. En Cobo Romaní, C. y Moravec J. (2011), Aprendizaje Invisible. Hacia una nueva ecología de la educación, Colección Transmedia XXI. Laboratori de Mitjans Interactius (pp. 159-182) Barcelona: Universitat de Barcelona.

Rinaudo, M. C. (2019). El estudio de la identidad en el campo de la Psicología Educacional. En P. V. Paoloni, M. C. Rinaudo y R. B. Martín, Yo, tu... ellos y nosotros. Competencias socioemocionales en la construcción de identidades profesionales (pp. 17-48). Córdoba: Editorial Brujas.

Salinas, J. (1997). Nuevos ambientes de aprendizaje para una sociedad de la información. Revista Pensamiento Educativo, 20.

Sanz Martos, S. (2005). Comunidades de práctica virtuales: acceso y uso de contenidos. En Lara Navarra, P. (coord.). Uso de contenidos digitales: tecnologías de la información, sociedad del conocimiento y universidad. Revista de Universidad y Sociedad del Conocimiento (RUSC), 2 (2).

Sarramona, J., Vázquez, G. y Colom, A. (1998), Educación no formal. Barcelona, España: Editorial Ariel Educación.

Seely Brown, J. (1999). Sustaining the Ecology of Knowledge, Leader to Leader, 12.

Schugurensky, D. (2000). The forms of informal learning. Towards a conceptualization of the field. Working Paper 19-2000, Presentado en New Approaches for Lifelong Learning (NALL) Fourth Annual Conference, 6-8 de Octubre. 
Scribano, A. (2017). Miradas cotidianas. El uso de Whatsapp como experiencia de investigación socia. Revista Latinoamericana de Metodología de la Investigación Social ReLMIS, (13), 7, 8-22.

Sequel Project: Quality Guide to the non-formal and informal Learning Processes (2004), Sustainable Environment for the Evaluation of Quality in E-Learning, ScienterMENON Network (pp 1-55).

Transliteracy (2018), Transmedialiteracy.org.

Trilla, J.; Gros, B.; López F. y Martín M. J. (2003), La educación fuera de la escuela. Ámbitos no formales y Educación Social. Barcelona, España: Ariel Educación.

Trilla, J. (1992). El sistema de educación no formal: Definición, conceptos básicos y ámbitos de aplicación. En Sarramona J. (ed.), La educación no formal (pp. 9-50). Barcelona, CEAC. 